\title{
Rheumatic Valvular lesions: Surgical Interventions
}

\author{
Koirala B., Koirala R., Sharma J., Pradhan S., Acharya A., Sharma P., \\ Rai K.,
}

This is a retrospective study of the patients with the diagnosis of Rheumatic Valvular Lesions, who had undergone surgical treatment at SGNHC between Aug 2001 and Jan 2004, The data was collected from the in-hospital records maintained for individual patient.

There were 320 patients operated for rheumatic valvular disease. There was female preponderance with male female ratio 1:1.5, The mean age was 30.1 (9-68yrs). Most of the patients $(90.6 \%)$ presented to us with the complaint of exertional dyspnoea. $74 \%$ of Patient were in NYHA III-IV category, $51 \%$ had atrial fibrillation. The echocardiography study revealed that the most common lesion was Mitral Stenosis (35\%), Mixed Mitral lesions (15\%), Mitral Regurgitation (5.6\%), Isolated Aortic Valve lesions (10.6\%) and rest were multiple valve disease (33.8\%). The mean LVIDd was 5.4 (3-10), mean LVIDs was $4.0(2-8)$ and the mean left atrial size was $5.3(2.3-9.5)$, There were 19 patients with left atrial/left atrial appendage clot.

The surgical procedures carried out were Closed Mitral Commisurotomy (27.2\%). Mitral valve Replacement (22.2\%). Aortic Valve Replacement (11.6\%). Double Valve Replacement with TV repair $(2.8 \%)$ and rest $(30.6 \%)$ had aortic or mitral valve repair/ replacement with tricuspid repair, The mean CPB time was 93,65min (28-360min) \& the mean aortic cross clamp time was $63.40 \mathrm{~min}(15-158 \mathrm{~min})$. The average postoperative bleed was $381.6 \mathrm{ml}$. 8 underwent re- exploration. The average stay in ICU was 2.1 days and post-operative stay was 7.9 days. $15 \%$ had varied post-operative complications like CVA, Chest infections, Organ Failure (Single or Multiple) \& Superficial Wound Infection.

95 patients had follow up at $3 \mathrm{mths}$ and there was remarkable improvement in cardiac size on echo with mean LVIDd-4.9(3.5-7.5), LVIDs-3.5(2.1 $\left.{ }^{\circ} 6.2\right)$, LA Size 4.5 (2.4-7.8). There were $12(3.8 \%)$ mortalities. 
- NEPALESE HEART JOURNAL

\section{Conclusion}

Valve lesions following post-rheumatic scarring not amenable to medical therapy due to progression of the disease can be aptly managed with surgical interventions, that can be individualized according to the type of valve lesion.

Department of Cardiovascular Surgery, SGNHC, Kathmandu, Nepal 